\title{
Fundamentos do Design para o Desenvolvimento de Tecnologia Assistiva
}

\author{
Design Fundamentals for Assistive Technology development \\ PRADO, Gheysa Caroline; Mestre; Universidade Federal do Paraná (UFPR) \\ gheysa.p@gmail.com
}

PELEGRINI, Alexandre Vieira; Doutor; Universidade Federal do Paraná (UFPR)

avpelegrini@yahoo.com

HEEMANN, Adriano; Doutor; Universidade Federal do Paraná (UFPR)

adriano.heemann@ufpr.br

\section{Resumo}

O presente artigo objetiva reconhecer as principais abordagens do design na tecnologia assistiva (TA) e identificar os principais autores da área de problemas complexos em design aplicado a TA. Utilizando uma Revisão Bibliográfica Sistemática (RBS), com roteiro de Conforto, Amaral e Silva (2011), coletar as principais lacunas de pesquisa apontadas pelos estudos existentes. Os resultados sugerem que a maioria dos trabalhos científicos sobre o assunto apontam ferramentas e técnicas de colaboração e co-design para desenvolvimento e sucesso dos dispositivos. Discute-se que apesar de consensos quanto ao desenvolvimento dos projetos para TA, há dificuldade na aplicação dessas técnicas e ferramentas.

Palavras Chave: design; tecnologia assistiva; problemas complexos.

\begin{abstract}
The present article aims to recognize the main design approaches in assistive technology (AT) and identify the main authors working on wicked problems in design for AT. Using a Systematic Literature Review (SLR), with Conforto, Amaral e Silva (2011) guide, detect the main research gaps identified by the existent studies. Results indicate most of scientific work about the subject are about collaborations and co-design tools and techniques for designing and success of the devices. We discuss that besides the consensus opinion on how to design for AT, there are some barriers on the use of these tools and techniques.
\end{abstract}

Keywords: design; assistive technology; wicked problems 


\section{GAMPI
plural ${ }^{15}$}

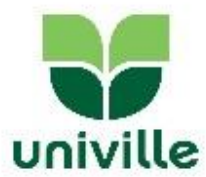

\section{Introdução}

Os estudos sobre o uso do design para o desenvolvimento de tecnologias assistivas (TA) sobretudo no que diz respeito aos métodos, técnicas e ferramentas projetuais tem crescido no meio acadêmico. Entretanto, os fundamentos do design para aplicação no desenvolvimento e inovação de TA para pessoas idosas no contexto de vida urbana ainda são bastante incipientes tendo em vista o aumento recente ${ }^{1}$ dessa necessidade e sua complexidade.

Este artigo descreve a aplicação de uma Revisão Bibliográfica Sistemática (RBS) preliminar cuja pergunta de pesquisa é: Quais são as ênfases e as lacunas de pesquisa na área do design para o desenvolvimento de TA para idosos no contexto urbano? Seus objetivos são: reconhecer as principais abordagens do design na área de TA; identificar os principais autores da área de problemas complexos em design aplicado a TA para pessoas idosas no contexto de vida urbana; e, como objetivo principal, identificar e coletar as principais ênfases e lacunas de pesquisa apontadas pelos estudos existentes.

A realização da RBS que possibilitou a identificação das ênfases e lacunas sobre o tema seguiu o roteiro proposto por Conforto, Amaral e Silva (2011) dividido em três fases: Entrada, Processamento e Saída. Os resultados mostraram a existência de literatura consolidada quanto à importância do uso de métodos, técnicas e ferramentas de design para o desenvolvimento de novas TA. Mostraram, também, a escassez de informações relativas ao público idoso no contexto de vida urbano.

A discussão aponta para a necessidade de aprofundamento na pesquisa sobre o uso desses métodos.

\section{Método}

Foi realizada uma RBS preliminar que permitiu perceber potenciais para novas pesquisas. Seu objetivo principal era identificar e coletar as principais ênfases e lacunas de pesquisa apontadas pelos estudos existentes nas áreas de: problemas complexos em design; e TA para pessoas idosas no contexto de vida urbano.

A realização da RBS de acordo com Conforto, Amaral e Silva (2011) e o seu relato em forma de artigo científico seguiram o cronograma apresentado no Quadro 1.

Para realização da presente RBS foi utilizado o roteiro desenvolvido por Conforto, Amaral e Silva (2011), composto por 15 etapas distribuídas em três fases: Entrada, Processamento e Saída. Na Entrada, definiu-se o problema da pesquisa: Quais são as ênfases e as lacunas de pesquisa na área do design para o desenvolvimento de TA para idosos no contexto urbano? Os objetivos a serem atingidos com a RBS: reconhecer as principais abordagens do design na área de TA; identificar os principais autores da área de problemas complexos em design aplicado a TA para pessoas idosas no contexto de vida urbano; e, como objetivo principal, identificar e coletar as principais ênfases e lacunas de pesquisa apontadas pelos estudos existentes.

\footnotetext{
1 "No Brasil, o número de idosos ( ${ }^{3} 60$ anos de idade) passou de 3 milhões em 1960, para 7 milhões em 1975 e 14 milhões em 2002 (um aumento de 500\% em quarenta anos) e estima-se que alcançará 32 milhões em 2020." (LIMA-COSTA e VERAS, 2003)
} 


\section{GAMPI plural ${ }^{45}$}

Quadro 1: Cronograma

\begin{tabular}{|c|c|c|c|c|c|c|c|c|c|c|}
\hline & \multicolumn{4}{|c|}{ Março } & \multicolumn{4}{|c|}{ Abril } & \multicolumn{2}{|r|}{ Maio } \\
\hline & 04 a 11 & 11 a 18 & 18 a 25 & 25 a 01 & 01 a 08 & 08 a 15 & 15 a 22 & 22 a 29 & 29 a 06 & 06 a 13 \\
\hline 1- Pressuposto: definição do tópico & $\mathrm{x}$ & & & & & & & & & \\
\hline 2- Revisão Bibliográfica Sistemática & & $x$ & $x$ & $x$ & $x$ & & & & & \\
\hline 3- Fichamento e coleta de figuras e exer & & & & $x$ & $\mathrm{x}$ & & & & & \\
\hline 4- Mapa conceitual e resumo preliminar & & & & & $x$ & $\mathrm{x}$ & & & & \\
\hline 5- Primeiro Rascunho & & & & & $\mathrm{x}$ & $\mathrm{x}$ & & & & \\
\hline 6- Detalhamento & & & & & & $\mathrm{x}$ & $\mathrm{x}$ & & & \\
\hline 7- Avaliação Prévia & & & & & & & & $\mathrm{x}$ & & \\
\hline 8- Reelaboração & & & & & & & & & $\mathrm{x}$ & \\
\hline 9- Maturação (pequenos ajustes) & & & & & & & & & $\mathrm{x}$ & \\
\hline 10- Preparação e Apresentação & & & & & & & & & & $x$ \\
\hline
\end{tabular}

Fonte: Arquivo Pessoal (2015)

Foram utilizadas três bases de dados, e os filtros foram aplicados de forma mais semelhante possível entre elas, com foco em "Periódicos revisados por pares", por assuntos relacionados e palavras-chave. Todas as buscas foram feitas utilizando os termos em língua inglesa.

\section{Resultado}

Como fontes primárias, foram identificadas algumas bases de dados, da seguinte forma: 1. "Buscar assunto" no Portal de Periódicos da CAPES, com a palavra "design" em primeiro, associando-a ao termo (AND) "assistive technology", filtro de "Periódicos revisados por pares". A busca retornou 618 artigos, a maioria, nas seguintes bases de dados: "OneFile (GALE)" e "SciVerse ScienceDirect (Elsevier)". A segunda busca na base da CAPES em "Buscar assunto", termos "design" AND "smart city", filtro de "Periódicos revisados por pares" retornou 95 artigos, com maioria dos artigos nas mesmas bases de dados mencionadas anteriormente. 2. Além das duas bases de dados apontadas pela pesquisa preliminar, era de conhecimento anterior da pesquisadora um terceira base de dados com indexação de um bom volume de referências de literatura revisada por pares, a Scopus (Elsevier). As mesmas palavras-chave utilizadas para identificação das fontes primárias foram utilizadas para formação dos strings de busca para realização da RBS, além da inclusão do termo "wicked problem" (problema complexo).

Como critérios de inclusão dos artigos, levou-se em conta o objetivo da revisão bibliográfica de identificar as ênfases e as lacunas de pesquisa na área do design para TA. Para tal, artigos que contivessem estudos sobre o desenvolvimento de produtos de TA no contexto urbano para pessoas idosas deveriam ser incluídos. Bem como aqueles que tratassem do uso da teoria de problemas complexos para processos de design.

Já como critério de qualificação, adotou-se a área da publicação do artigo, que deve estar dentro da área de interesse da pesquisa, design (e suas áreas de inserção que variam de local para local - ciências sociais e humanidades; arquitetura, urbanismo e design etc.) e TA, incluindo, Design Universal ambos no contexto urbano, podendo aceitar áreas adjacentes como a ergonomia e a sustentabilidade, por exemplo. Como não foi apontado nenhum intervalo de tempo para a busca, a data da publicação foi usada como critério de qualificação 


\section{GAMPI
plural'15}

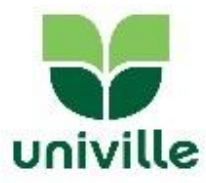

para discrepância de data dos demais artigos encontrados. Exceto para o caso de literatura de base, reconhecidamente importantes para a área pesquisada.

Para a realização da busca nas bases de dados, usando os strings mencionados anteriormente, para cada base consultada, seguiu-se a mesma ordem sistemática de busca por palavras-chave. As combinações seguiram a seguinte ordem: 1. "design AND 'assistive technology"'; 2. "design AND 'wicked problems"', strings associados, ou não, a outros como "city" e "smart city"

No Processamento, foram realizadas as buscas e seleção conforme segue:

Em OneFile (GALE) a busca teve teve a seguinte sequência: 1. "design AND 'assistive technology"' em "Keyword (Academic Journals)", com número de resultados de 285 artigos. 1.1. Filtro "Peer Reviewed Journals" e "Article", redução para 62 artigos. 1.2. Seleção por assunto "Assistive technology devices" resultou em 22 artigos dos quais, após seleção por título e resumos de acordo com os critérios de inclusão e qualificação apontados, teve 3 artigos mantidos para leitura de introdução e conclusão. 1.3. Seleção por assunto "the elderly", nos mesmos 62 artigos, obteve-se 6 artigos como resultado, dos quais 2 permaneceram após seleção por título e resumos. 2. "design AND 'wicked problems"' em "Keyword (Academic Journals)", 27 resultados. 2.1 Filtro "Peer Reviewed Journals" e "Article", 8 artigos, dos quais 2 permaneceram após seleção por título e resumos. 3. "design AND 'smart city"' em "Keyword (Academic Journals)", 15 resultados. 3.1 Filtro "Peer Reviewed Journals" e "Article", 6 artigos, permanecendo 1 após seleção por título e resumos. Buscas com mais de 2 palavras-chave nessa base de dados não trouxeram nenhum resultado. No total, 8 artigos foram selecionados em OneFile (GALE).

$\mathrm{Na}$ base de dados "ScienceDirect" a busca foi realizada sempre em "Title, Abstract, Keyword (Article)" e teve a seguinte sequência: 1. "design AND 'assistive technology"', com 124 resultados. 1.1 Filtro "Topic: 'assistive technology", 10 artigos, dos quais 3 permaneceram após seleção por título e resumos. 1.2 Filtro "Topic: 'environmental factor"', 2 artigos, 1 após seleção. 2. "design AND "assistive technology" AND city", 4 artigos, nenhum filtro foi aplicado e nenhum artigo permaneceu após seleção. 3. "design AND "assistive technology" AND problem", 17 resultados, nenhum filtro foi aplicado e 1 artigo permaneceu após seleção. 4. "design AND 'wicked problems"', 15 resultados, nenhum filtro foi aplicado e 5 artigos permaneceram após seleção. 5. "design AND 'smart city' OR 'smart cities"', 21 resultados, nenhum filtro foi aplicado, 4 artigos permaneceram após seleção. No total, 15 artigos foram selecionados na ScienceDirect.

$\mathrm{Na}$ base de dados "Scopus", a busca foi realizada sempre em "Article Title, Abstract and Keyword" e teve a seguinte sequência: 1. "( design AND "assistive technology" AND DOCTYPE(ar))", 930 resultados. 2. "design AND "assistive technology" AND city OR cities AND DOCTYPE ( ar )", 10 documentos, 1 permaneceu após seleção por título e resumos. 3. "( design AND "assistive technology" ) AND DOCTYPE ( ar ) ) AND ( ( city OR cities ) ) AND ( urban environment )", 6 artigos, 2 permaneceram após seleção. 4. "( design AND "assistive technology") AND DOCTYPE ( ar ))) AND ("wicked problem" OR "wicked problems" )", 4 documentos, 2 permaneceram. No total, 5 artigos foram selecionados na Scopus.

Tendo selecionado 28 artigos de Saída, após a releitura de Títulos, Resumos e PalavrasChave, 13 artigos foram selecionados para leitura completa. Dos 13 artigos selecionados. 3 não estavam disponíveis para além do resumo e, 2 se mostraram irrelevantes para a pesquisa. 


\section{GAMPI
plural ${ }^{15}$}

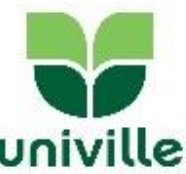

A fase de Saída, para este artigo, está na identificação das ênfases e lacunas, por meio da síntese dos resultados encontrados nos 8 artigos selecionados e apresentados nas próximas seções.

\section{Discussão}

Através da realização da presente RBS é possível sugerir que o design aliado à TA é recorrente na literatura. Dos 8 artigos utilizados, 5 autores, Laviolette e Hanson (2007), De Couvreur e Goossens (2011), Greenhalgh et. al. (2013), Herriot (2013) e Saborowski e Kollak (2014) apontam não somente a importância do design no desenvolvimento dessas tecnologias como também o uso de métodos, técnicas e ferramentas de design centrado no usuário envolvendo colaboração e co-design para tal. Um termo que aparece com frequência, associado à TA é o Design Universal sendo tratado, nessa RBS, por De Couvreur e Goossens (2011) e Plos et. al. (2012). De Couvreur e Goossens (2011) tratam ainda da existência de componentes complexos para toda e qualquer questão de design. Os problemas complexos, apresentados pela primeira vez por Rittel e Webber (1973), foram revisitados por Coyne (2005) que reforça a formulação complexa para problemas de design como regra e não exceção.

Partindo do conceito de maior amplitude, De Couvreur e Goossens (2011) descrevem o Design Universal como uma prática que cresceu a partir de uma demanda legal, de "adaptação ambiental necessária para acomodar pessoas com necessidades especiais" para a "remoção de barreiras (...) para integração de todas as pessoas em todos os ambientes". Evoluiu ainda mais, segundo os autores, tornando-se uma prática geral de design em projetar de forma a atender as necessidades do maior número de pessoas possível, independente de idade ou habilidade (MACE, 1985 apud DE CONVREUR e GOOSSENS, 2012).

Enquanto Laviolette e Hanson (2007), Greenhalgh et. al. (2013) e e Saborowski e Kollak (2014) afirmam que os estudos para o desenvolvimento de inovações na área de TA ainda negligenciam as percepções dos usuários acerca de suas próprias necessidades, sendo muitas vezes ainda distantes de atender a realidade do dia-a-dia das pessoas. Plos et. al. (2012) contrapõem afirmando que os produtos de TA, são altamente especializados e específicos, tendendo a um mercado de nicho, o que não seria bom do ponto de vista da produção dos mesmos. Plos et. al. (2012) propõem, então, um modelo que tem como objetivo estimular a inovação no campo de TA, melhorando o design dos produtos de forma a tornálos mais universais, conciliando a visão especializada com a universal.

Seguindo a visão de De Couvreur e Goossens (2011) em oposição a Plos et. al. (2012), a TA é uma área de necessidade de desenvolvimento e atuação praticamente individuais, na qual soluções locais são mais efetivas por refletirem as necessidades físicas, cognitivas e emocionais dos usuários. Para tanto, descrevem um modelo de desenvolvimento colaborativo e horizontal em uma rede de inovação integrada de e para usuários com deficiência, composta por designers, terapeutas ocupacionais e usuários. Para os autores, há mais oportunidades de design no mundo - especialmente nessa área - do que designers sozinhos podem resolver.

Diferentemente do apontado por Plos et. al. (2012), De Couvreur e Goossens (2011) enfatizam que na área de TA não há um "usuário médio" vivendo em um "contexto médio". Portanto, a solução de problemas para esse público, segundo os autores, tem um certo "componente complexo". A necessidade de desenvolvimento nessa área e o envolvimento dos usuários é reforçado por Greenhalgh et. al. (2013) e Saborowski e Kollak (2014) que também 


\section{GAMPI}

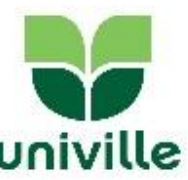

tratam da importância do envolvimento não apenas dos usuários, mas dos terapeutas ocupacionais junto aos designers nos projetos de TA.

Conforme citado anteriormente, há um grande número de métodos, técnicas e ferramentas disponíveis para tal envolvimento dos usuários no desenvolvimento de TA. No entanto, Herriott (2013) discorre sobre a dificuldade de aplicação destes métodos, técnicas e ferramentas para participação do usuário em projetos de TA realizados por designers. Em um levantamento e análise de 66 projetos de design para TA, Herriot (2013) relaciona uma lista de 6 etapas de projeto que deveriam ser cumpridas para o design de TA. Constatou, no entanto, a baixa adesão dos designers a todas a etapas, o que, segundo o autor, leva a um desaparecimento das demandas iniciais dos usuários ao longo do projeto, reforçando a ideia do "componente complexo" para o projeto de TA apresentada por De Couvreur e Goossens (2011).

Acerca do componente complexo, sugerido por De Couvreur e Goossens (2011), Coyne (2005), baseado em Rittel e Webber (1973), afirma que as tarefas do design são invariavelmente complexas. Segundo Coyne (2005) as soluções apresentadas estão ligadas ao local e contexto onde serão aplicadas não podendo, normalmente, após testadas, serem desfeitas. O autor argumenta ainda que, no design, o complexo é o normal e que formulações simples de problemas são o desvio à regra.

Em 1973, Rittel e Webber, um professor de design e outro de planejamento urbano definiram como problemas complexos a inexistência de bases científicas para tratar de políticas e problemas sociais. Segundo os autores, a ciência só está acostumada a trabalhar com problemas simples, os quais possuem formulação passível de resposta. Para eles, em uma sociedade pluralista, não faz sentido falar em soluções ótimas e, para questões sociais, não haveria nem mesmo o verdadeiro e o falso. Rittel e Webber (1973) levantam a questão, inclusive, da inexistência de solução possível, do ponto de vista de respostas definitivas e objetivas para um problema.

O tema e a definição de problemas complexos não é novidade no campo do design, como se verifica em Rittel e Webber (1973), assim como não o é a TA (MACE, 1985 apud DE CONVREUR e GOOSSENS, 2012). A associação das três áreas, entretanto, parece ainda incipiente e passível de investigação mais aprofundada.

Apesar de alguma oposição, apresentada por Plos et. al. (2012) que afirma que a TA deve ser mais universalizada de forma a atender melhor a lógica de mercado, é quase consenso entre os autores Laviolette e Hanson (2007), De Couvreur e Goossens (2011), Greenhalgh et. al. (2013), Herriot (2013) e Saborowski e Kollak (2014) que o uso de colaboração, co-design e até mesmo personalização deve ser mandatório para o desenvolvimento e sucesso de TA. Greenhalgh et. al. (2013) vão além, ao afirmar que boa parte dos dispositivos de TA existentes atendem pouco as necessidades dos usuários. Segundo os autores, os dispositivos que obtiveram algum sucesso no seu uso e assistência sofreram, necessariamente, alguma modificação e/ou customização pelos usuários ou por pessoas responsáveis pelos seus cuidados - terapeutas ocupacionais ou familiares.

Face a essa necessidade Herriot (2013) investigou, por meio de revisão de literatura, o desenvolvimento de 66 projetos de design para TA, do ponto de vista da metodologia projetual e constatou o baixo envolvimento e adesão dos designers a metodologias de design centrado no usuário ao longo de todo o processo. Segundo Herriot (2013), apenas 4,5\% dos 


\section{GAMPI plural 15}

projetos seguiram todas as etapas de desenvolvimento e, 39,3\% reportaram ter realizado apenas 1 das 6 etapas por ele descritas.

Sabe-se, e Coyne (2005) e De Couvreur e Goossens (2011) reforçam a teoria, que problemas de design são problemas de formulação complexa. Não há uma única solução, pois tudo depende de quem está fazendo a leitura do problema, dos usuários, e do contexto em que ele está inserido. Rittel e Webber (1973), e a releitura feita por Coyne (2005), permitem entender que, muitas vezes, nem mesmo há solução.

\section{Conclusão}

Muitos trabalhos conceituados no campo do design abordam os fundamentos do design aplicado à TA. Através desta RBS, 8 artigos foram selecionados e permitiram reconhecer as principais abordagens do design na área de TA como sendo em diversos métodos, técnicas e ferramentas para o desenvolvimento de novos dispositivos. A aplicação desses recursos, entretanto, como pode ser verificado em Herriot (2013) ainda é insipiente e os designers encontram dificuldades em fazê-lo.

Por meio dessa pesquisa é possível sugerir que a literatura atual e os principais autores da área incentivam a colaboração e o co-design no desenvolvimento de TA por meio de métodos, técnicas e ferramentas de design centrado no usuário, nesse caso, as pessoas idosas. Entretanto, ainda não há suficientes evidências de que esses tenham sido usados de forma efetiva no desenvolvimento das TA existentes. Pelo contrário, Greenhalgh et. al. (2013), por exemplo, afirmam que o sucesso da maior parte das TA por eles pesquisadas se deve quase que exclusivamente à intervenção realizada pelos usuários ou cuidadores do que pelo projeto assertivo.

Dentre as lacunas apresentadas pelas pesquisas, está a de que os dispositivos de TA para idosos existentes hoje no mercado estão longe de considerar adequadamente a demanda dos usuários principalmente no contexto de vida urbana, conforme demonstrado em Greenhalgh et. al. (2013) e Laviolette e Hanson (2007). Os métodos, técnicas e ferramentas existentes (COYNE, 2005; DE COUVREUR e GOOSSENS, 2011 e HARRIOT, 2013), apesar de incentivarem a colaboração e o co-design, ainda apresentam problemas de uso e aplicação.

Diante deste panorama, recomenda-se que pesquisas futuras busquem a identificação e a compreensão dos motivos que fazem designers preferirem abandonar os métodos, técnicas e ferramentas existentes para o desenvolvimento de TA. Também pode-se sugerir uma investigação sobre o uso da teoria de problemas complexos no campo do design para TA e os impactos positivos e negativos que poderia causar.

\section{Agradecimento}

Os autores agradecem pelo apoio Fundação Araucária do Paraná.

\section{Referências}

CONFORTO, E. C.; AMARAL, D. C.; SILVA, S. L. DA. Roteiro para revisão bibliográfica sistemática: aplicação no desenvolvimento de produtos e gerenciamento de projetos. $\mathbf{8}^{\circ}$ Congresso Brasileiro de Gestão de Desenvolvimento de Produto - CNGDP 2011, p. 1-12, 2011. 


\section{GAMPI plural| 15}

COYNE, R. Wicked problems revisited. Design Studies, v. 26, n. 1, p. 5-17, 2005.

DE COUVREUR, L.; GOOSSENS, R. Design for (every)one: Co-Creation As a Bridge Between Universal Design and Rehabilitation Engineering. CoDesign, v. 7, n. 2, p. 107-121, 2011.

GREENHALGH, T. et al. What matters to older people with assisted living needs? A phenomenological analysis of the use and non-use of telehealth and telecare. Social Science and Medicine, v. 93, p. 86-94, 2013.

GRUPO STELA. Guia de preparação de artigos. Florianópolis: UFSC, 2004. Disponível em: $<$ http://www.labcon.ufsc.br/downloads/33.pdf>. Acesso em: 04 mar. 2015.

HERRIOTT, R. Are inclusive designers designing inclusively? An analysis of 66 design cases. Design Journal, v. 16, n. 2, p. 138-158, 2013.

LAVIOLETTE, P.; HANSON, J. Home_is_where_the_heart_stopped. Home Cultures, v. 4, n. 1, p. 25-44, 2007.

LIMA-COSTA, Maria Fernanda; VERAS, Renato. Saúde pública e envelhecimento. Cad. Saúde Pública, Rio de Janeiro, v. 19, n. 3, May 2003.

PLOS, O. et al. A Universalist strategy for the design of Assistive Technology. International Journal of Industrial Ergonomics, v. 42, n. 6, p. 533-541, 2012.

RITTEL, H. W. J.; WEBBER, M. M. Dilemnas in a general theory of planning. Policy Sciences, v. 4, n. December 1969, p. 155-169, 1973.

SABOROWSKI, M.; KOLLAK, I. "How do you care for technology?" - Care professionals' experiences with assistive technology in care of the elderly. Technological Forecasting \& Social Change, v. 93, p. 133-140, 2014. 